\title{
El pian en la provincia de Esmeraldas, Ecuador
}

\author{
Ronald H. Guderian', Mariela Anselmi², Manuel Calvopiña ${ }^{3}$, Philip J. Cooper ${ }^{3}$, Tamara Mancero ${ }^{3}$
}

\section{Resumen}

Se realizó un estudio epidemiológico, clínico y serológico del pian en la cuenca del río Santiago, cantón Eloy Alfaro, provincia de Esmeraldas, el cual demostró una prevalencia del $11,3 \%$ con lesiones dérmicas activas y una seropositividad del $94,9 \%$. El área del río Santiago era hiperendémica para el pian (prevalencia 17,9\%; seropositividad $98,0 \%$ ), mientras que el río Zapallito era mesoendémico y los ríos Cayapas y Onzole, hipoendémicos. Se encontró evidencia del pian, activa y latente, solamente en la raza negra. La mayor incidencia de las lesiones clínicas $(55,8 \%)$ se encontró en los niños de 5-12 años de edad, localizándose el porcentaje superior en las piernas $(64,2 \%)$. Los papilomas fueron las lesiones más frecuentes $(55,4 \%)$. Se administró tratamiento masivo con penicilina benzatínica, según las normas de OMS, en todas las comunidades positivas para esta enfermedad.

\section{Summary}

A clinical and serological epidemiological study in a yaws focus in cantón Eloy Alfaro, in the Santiago basin area of the province of Esmeraldas, showed a prevalence of $11.3 \%$ for active skin lesions and seropositivity of $94.9 \%$. The Santiago river was found to be hyperendemic for yaws (prevalence 17.9), while the Zapallito river was mesoendemic and the Cayapas and Onzole rivers were hypoendemic. Active and latent evidence of yaws was found only in the black race. Children, aged 5-12, were found to have the highest incidence $(55.8 \%)$ of clinical lesions, the majority being found on the legs $(64.2 \%)$. The most prevalent lesions $(55.4 \%)$ were papilloma. Mass treatment of all positive communities, using benzathine penicillin $\mathrm{G}$, was carried out according to $\mathrm{WHO}$ recommendations.

El pian, una enfermedad contagiosa no venérea producida por Treponema pertenue, también llamada buba, bouba o frambesia, está virtualmente confinada a las poblaciones tropicales primitivas de las áreas rurales en los trópicos mediogeográficos. La enfermedad es endémica a

1 Médico investigador, Director, Departamento de Investigaciones Clínicas, Hospital Vozandes, Quito, Ecuador.

2 Médica investigadora, Salud Primaria Comunitaria, Vicariato Apostólico Esmeraldas, Provincia de Esmeraldas.

3 Médico investigador, Departamento de Investigaciones, Hospital Vozandes, Quito, Ecuador. lo largo de todo el Africa tropical, Tailandia, Malasia, Indonesia, Filipinas, Suramérica y las islas del $\mathrm{Ca}$ ribe (1).

En 1959, se estimaba que la incidencia mundial era de aproximadamente 50 millones de casos y en algunos países se tuvo la sospecha que existían 20 a 30 casos por cada caso informado en las estadísticas oficiales.

En la década de los cincuenta, con auspicio de organizaciones internacionales como la OMS y la UNICEF, se realizaron programas sistemáticos para la erradicación del pian en todos los países, obteniéndose una disminución significativa documentada mundialmente. 
Desde 1983, en Latinoamérica se informaron casos esporádicos en áreas geográficas limitadas a Brasil, Colombia, Guayana y Surinam (2).

En el Ecuador, el foco principal del pian se encuentra en la cuenca del río Santiago, provincia de Esmeraldas (3). Aunque, desde el año 1942 había algunos programas nacionales para su erradicación (4), el éxito alcanzado inicialmente fue seguido de la suspensión de los programas y el pian volvió y se convirtió nuevamente en un problema de salud pública $(5,6)$. Recientemente se realizó un estudio epidemiológico, clínico y serológico en la cuenca del río Santiago para determinar la situación actual de la enfermedad e iniciar un programa de control.

\section{Materiales y métodos}

Se realizó un estudio epidemiológico, clínico y serológico seguido de una campaña de tratamiento para el pian en la cuenca del río Santiago, provincia de Esmeraldas. Esta área está formada por la unión de tres ríos principales y sus tributarios: río Santiago, río Onzole y río Cayapas (7) (figura 1).

Se estudiaron 87 comunidades localizadas en esta área, con 2.963 individuos examinados, lo cual representa el $93 \%$ de la población según el censo actualizado.

La población del área de estudio está formada por dos razas puras; la negra, con ancestros africanos y, una tribu indígena, los Chachi. Las características raciales, culturales, geográficas y climáticas de esta zona ya han sido descritas anteriormente (8).

Se realizó el estudio epidemiológico con base en un censo actualizado de todas las comunidades, registrando a los que presentaban variadas lesiones dérmicas del pian. En un formulario preparado para el estudio, se anotaron todos los datos pertinentes a la investigación como raza, sexo, edad, sitio de la lesión, tipo de la lesión, duración y fecha del tratamiento. A cada individuo, se le examinó toda la superficie dérmica y el diagnóstico del pian fue hecho según la apariencia de las lesiones dérmicas (9).

Se realizó una encuesta serológica, utilizando la prueba RPR (10) en todos los moradores que tenían más de 5 años de edad en las comunidades investigadas para lesiones dérmicas del pian. Todas las que salieron positivas para RPR se confirmaron con la prueba de FTA-Abs.

Se determinó la taza de endemicidad de la enfermedad según los criterios recomendados por la OMS (11). Para considerar a una región geográfica como endémica debería presentar una prevalencia de lesiones superior al $10 \%$ y una seropositividad superior al $90 \%$.

El tratamiento se administró según las recomendaciones de la OMS (12). La penicilina benzatínica se aplicó a todas las personas que presentaban lesiones dérmicas del pian. En las comunidades donde se encontró una seropositividad de RPR superior al $80 \%$, se realizó el tratamiento masivo. Después de dos semanas, se efectuó un control de los casos con lesiones activas para evaluar la eficacia del tratamiento.

\section{Resultados}

Area endémica: de las 87 comunidades examinadas a lo largo de los ríos Santiago, Cayapas y Onzole, $20(23,0 \%)$, salieron positivas para el pian. De las 2.964 personas que radicaban en las 20 comunidades, $333(11,2 \%)$ presentaron clínicamente lesiones dérmicas activas del pian. En las 20 comunidades hubo una seropositividad del $93,6 \%$. La seropositividad en las comunidades en donde no se encontraron lesiones activas dérmicas del pian era del $72,5 \%$.

Se clasificaron las comunidades positivas para el pian en cuatro focos (figura 2). El principal se encontró a lo largo del río Santiago, desde la comunidad Playa de Oro hasta Rocafuerte. Otros focos pequeños se encontraron en el río Cayapas, formado por las comunidades de San Miguel hasta Telembí; en el río Zapallito por las comunidades de Juan Montalvo y Aquí Me Quedo; y en el río Onzole por las comunidades del Colón y Santo Domingo. 


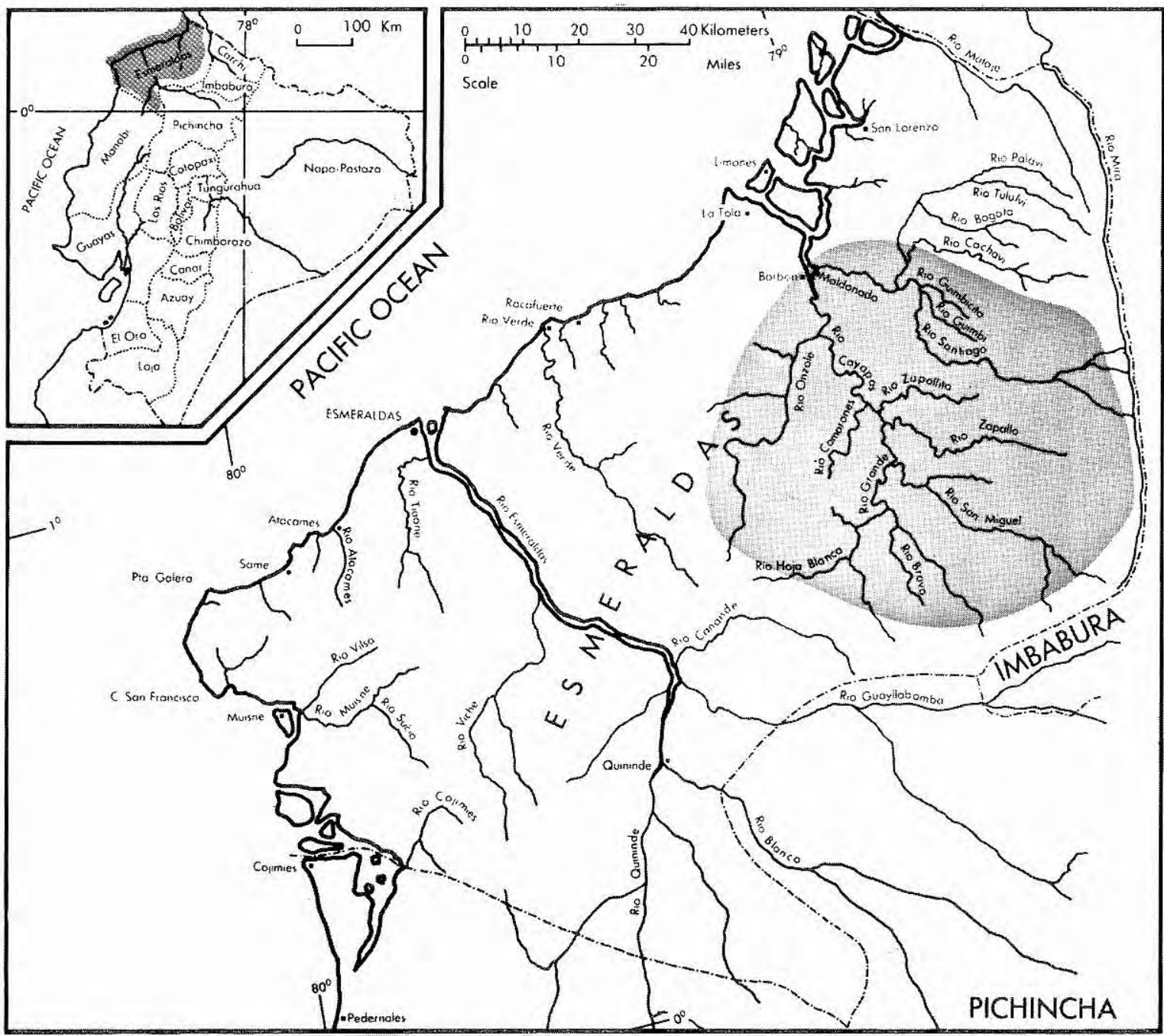

Figura 1. La cuenca del río Santiago, cantón Eloy Alfaro, provincia de Esmeraldas, Ecuador, sitio endémico para el pian.

Prevalencia: la prevalencia del pian en los cuatro focos se encuentra en la tabla 1. El río Santiago, según la clasificación de la OMS, era un área endémica para el pian con una prevalencia de lesiones activas dérmicas del 17,9\% y con una seropositividad de $98,0 \%$. Las comunidades más positivas eran Rocafuerte (26,7\%) y SelvaAlegre $(23,0 \%)$. La prevalencia en los otros focos que se encontraron en los ríos Cayapas, Zapallito y Onzole eran menores $(4,6 \%, 4,6 \%$ y $1,7 \%$, respectivamente) aunque la seropositividad se mantuvo elevada.
La distribución por grupos etáreos con lesiones dérmicas del pian se encuentra en la figura 3. Las personas menores de 19 años de edad presentaron el mayor porcentaje de lesiones $(84,7 \%)$. La prevalencia más alta fue en los que tenían 5 a 12 años de edad $(55,8 \%)$. El sexo masculino presentó con mayor frecuencia las lesiones dérmicas $(63,4 \%)$ que el sexo femenino $(36,6 \%)$. En todas las comunidades estudiadas, se encontraron solamente los individuos de la raza negra positivos para el pian. 


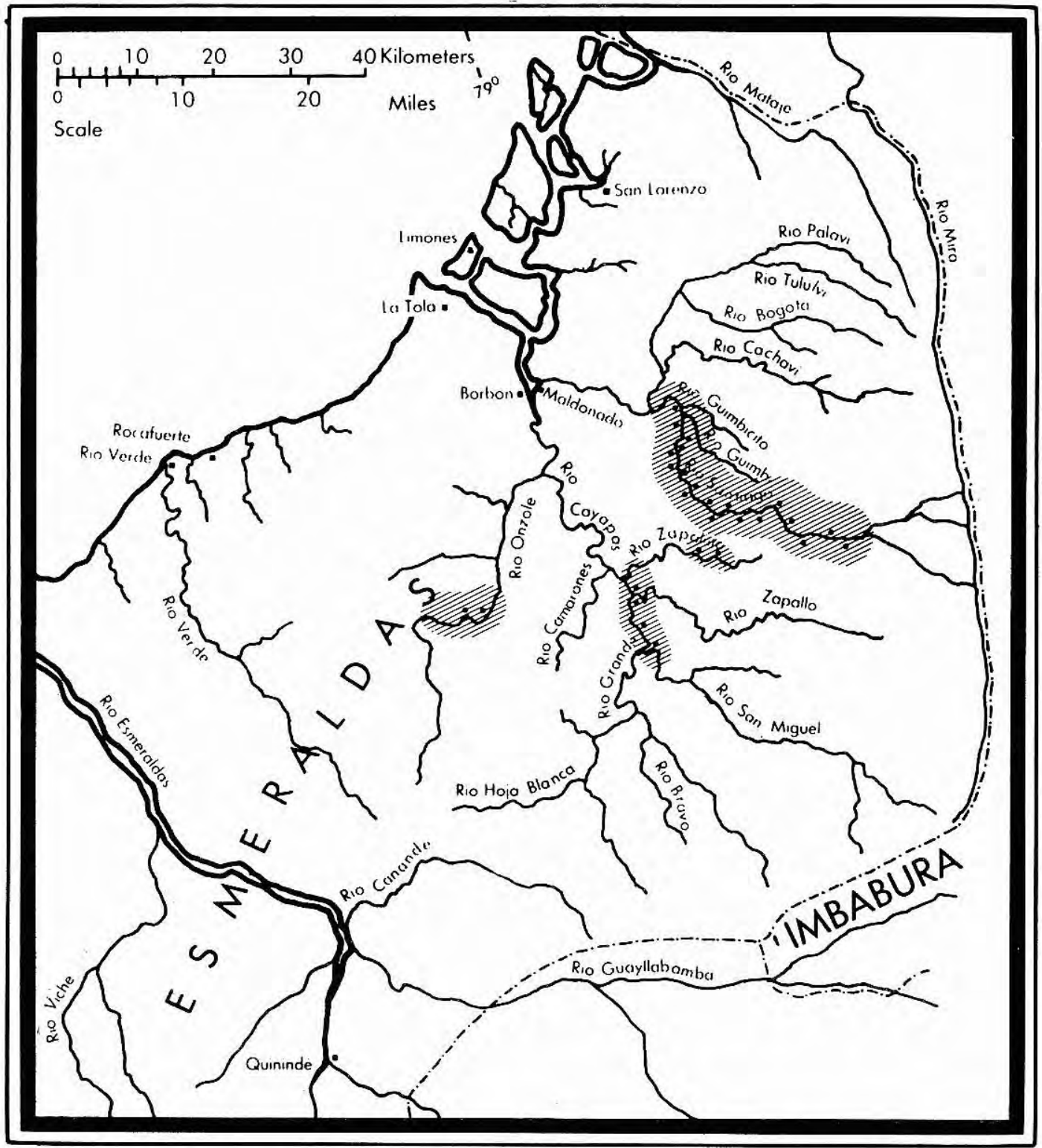

Figura 2. Los cuatro focos con sus respectivas comunidades positivas para el pian en la cuenca del río Santiago, provincia de Esmeraldas.

Manifestaciones clínicas: las lesiones del pian pueden manifestarse clínicamente en tres fases diferentes: primaria, secundaria y tardía. Se encontraron las lesiones en las tres fases diferentes en el foco ecuatoriano (figura 4). Las lesiones de la fase primaria, los papilomas y las úlceras, se encontraron con más frecuencia, $52,4 \%$ y $25,4 \%$, respectivamente. Las lesiones de la fase secundaria, las verrugas e hiper- queratosis de la planta del pie, se presentaron con menos frecuencia, $9,5 \%$ y $4,8 \%$, respectivamente. La lesión tardía, la gangosa, se encontró solamente en el río Cayapas con una prevalencia del $2,9 \%$.

La distribución anatómica de las lesiones dérmicas se encuentra en la figura 5 . Se presentaron con mayor frecuencia en las piernas $(62,4 \%)$, seguidas por los brazos $(13,7 \%)$, los 
Tabla 1. Prevalencia de las lesiones clínicas y la seropositividad en las comunidades positivas para el pian en la provincia de Esmeraldas, Ecuador.

\begin{tabular}{|c|c|c|c|c|c|}
\hline \multirow{3}{*}{$\begin{array}{c}\text { Sitio } \\
\text { geográfico }\end{array}$} & \multirow{3}{*}{$\begin{array}{l}\text { Examinado } \\
\mathbf{n}\end{array}$} & \multicolumn{4}{|c|}{ Prevalencia } \\
\hline & & \multicolumn{2}{|c|}{ Lesiones } & \multirow[b]{2}{*}{. } & Serología \\
\hline & & $\mathbf{n}$ & $\%$ & & $\%$ \\
\hline \multicolumn{6}{|l|}{ Río Santiago } \\
\hline Playa de Oro & 120 & 12 & 10,0 & 118 & 98,3 \\
\hline Angostura & 30 & 6 & 20,0 & 29 & 96,6 \\
\hline Playa Tigre & 88 & 17 & 19,3 & 86 & 97,7 \\
\hline Guayabal & 180 & 38 & 21,1 & 173 & 96,1 \\
\hline Chanazal & 66 & 10 & 15,2 & 65 & 98,5 \\
\hline Selva Alegre & 283 & 65 & 23,0 & 282 & 99,6 \\
\hline Timbiré & 222 & 28 & 12,6 & 218 & 98,2 \\
\hline Porvenir & 81 & 18 & 22,2 & 79 & 97,5 \\
\hline Las Antonias & 169 & 22 & 13,0 & 166 & 98,2 \\
\hline Negrital & 101 & 20 & 19,8 & 99 & 98,0 \\
\hline La Peña & 189 & 29 & 15,3 & 184 & 97,3 \\
\hline Rocafuerte & 101 & 27 & 26,7 & 98 & 97,0 \\
\hline Total & 1.630 & 282 & 17,9 & 1.597 & 98,0 \\
\hline \multicolumn{6}{|l|}{ Río Cayapas } \\
\hline San Miguel & 42 & 8 & 19,0 & 40 & 95,2 \\
\hline Mafua & 98 & 2 & 2,0 & 80 & 81,6 \\
\hline Zapallo Grande & 101 & 4 & 4,0 & 83 & 82,2 \\
\hline Telembí & 282 & 10 & 3,5 & 246 & 87,2 \\
\hline Total & 523 & 24 & 4,6 & 449 & 86,6 \\
\hline \multicolumn{6}{|l|}{ Río Zapallito } \\
\hline Juan Montalvo & 124 & 11 & 8,9 & 115 & 92,7 \\
\hline Aquí me Quedo & 103 & 7 & 6,8 & 93 & 90,3 \\
\hline Total & 227 & 24 & 4,6 & 208 & 91,6 \\
\hline \multicolumn{6}{|l|}{ Río Onzole } \\
\hline Colón & 293 & 6 & 2,0 & 285 & 97,3 \\
\hline Santo Domingo & 291 & 4 & 1,4 & 277 & 95,2 \\
\hline Total & 584 & 10 & 1,7 & 562 & 96,2 \\
\hline
\end{tabular}

pies $(6,9 \%)$, la cara $(5,4 \%)$, la espalda $(3,4 \%)$, la cadera $(2,9 \%)$, el abdomen $(2,0 \%)$ y el pene $(1,5 \%)$. La mayoría de los individuos positivos para el pian presentaban lesiones múltiples (73,2\%).

\section{Tratamiento}

De acuerdo con la recomendación de la OMS de que en un área endémica para el pian, toda la población debería ser tratada, todas las comunidades en el río Santiago fueron tratadas con penicilina benzatínica en dosis de acuerdo con la edad. En los ríos Cayapas, Zapallito y Onzole solamente las comunidades con lesiones del pian y una seropositividad superior al
$80 \%$ fueron tratadas. Las comunidades $\sin$ lesiones del pian y una seropositividad menor al $80 \%$ no recibieron ningún tratamiento. En $98,3 \%$ de los casos con lesiones dérmicas activas hubo una curación completa. Con las lesiones que no sanaron, se realizaron otros estudios para determinar la causa de la resistencia.

\section{Discusión}

En el Ecuador, desde la década de los 40 , se han encontrado casos de pian en la zona norte de la provincia de Esmeraldas y en el oriente (3). Aunque la mayoría de los casos provenían de Esmeraldas, también se informaron casos 


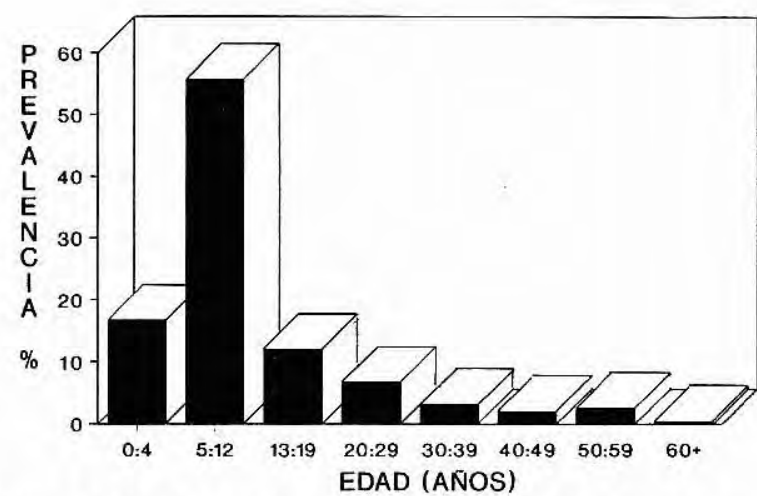

Figura 3. La distribución etárea de los individuos positivos para el pian en la cuenca del río Santiago, provincia de Esmeraldas.

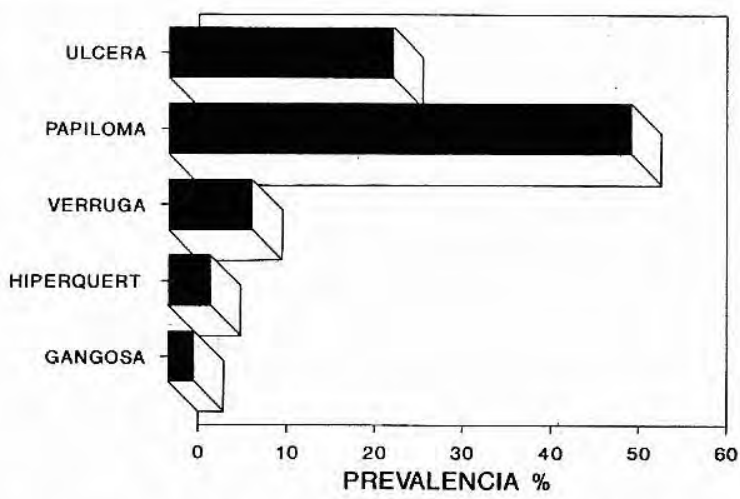

Figura 4. Prevalencia de los tipos de la lesiones del pian encontrados en individuos positivo en la cuenca del río Santiago, provincia de Esmeraldas.

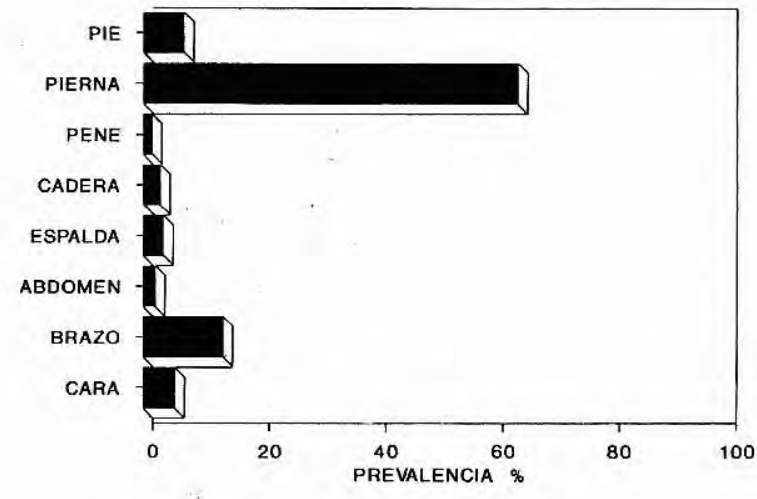

Figura 5. Distribución anatómica de las lesiones encontras en individuos positivos para el pian en la cuenca del río Santiago, provincia de Esmeraldas. en Cotundo, Archidona y sus alrededores en la provincia del Napo $(13,14)$. Hasta el año de 1942, el control de esta enfermedad se hacía en forma irregular, sin una orientación definida. En 1950, el Servicio Cooperativo Interamericano de Salud Pública organizó una campaña dirigida especialmente al control endémico utilizando para ello penicilina, lo cual alteraba sustancialmente el tratamiento, pues se suspendió el tratamiento seriado con arsenicales.

En 1958, a través de la campaña de erradicación del pian del SNEM, se efectuaba un tratamiento masivo en las áreas de mayor incidencia de la enfermedad, continuándose la vigilancia hasta 1964 cuando con una incidencia del 0,03 x 1000 habitantes, se entregó la campaña al Ministerio de Previsión Social, Trabajo y Salud, haciendo constar la necesidad de mantener una vigilancia epidemiológica por el peligro de una reinfección.

Estas recomendaciones no se cumplieron y en 1968 se presentó un aumento significativo de casos, especialmente en el área norte de la provincia de Esmeraldas. A comienzos de 1972, el SNEM realizó una encuesta para conocer la magnitud del problema, comprobándose la existencia de 466 casos dispersos en localidades del cantón Eloy Alfaro. En octubre de 1974, un control del programa demostró que existía la necesidad de intensificar las acciones de tratamiento y control de contactos.

En 1975, en el mes de noviembre, se realizó una evaluación en tres sectores diferentes en la zona norte de la provincia de Esmeraldas. Los resultados demostraron que se trataron los casos positivos en un $96,7 \%$, con sólo un $47 \%$ de cobertura de los contactos. Sin embargo, en los últimos 18 años no ha habido continuidad en los programas nacionales para el control del pian, con la correspondiente elevación de tasas de reinfección.

Es interesante anotar que desde los años de 1940, ningún caso de pian ha sido reportado desde el oriente. Esto nos hace pensar que probablemente la enfermedad fue erradicada en esta época por los programas de control realizados. La razón por la cual todavía existe 
el foco en la provincia de Esmeraldas indica una continuación con el foco colombiano que se encuentra en el departamento de Nariño (15) y, por el intercambio que existe entre los dos países, existe la posibilidad de que la enfermedad fue reintroducida al Ecuador.

Las manifestaciones del pian en la cuenca del río Santiago eran consistentes con las que se han informado en otras partes del mundo $(9,12)$. Es una enfermedad prominentemente de zonas rurales, calientes, húmedas y tropicales. En el Ecuador, la mayor incidencia de la enfermedad se encontró en los que tenían menos de 15 años de edad, con los niños más infectados que las niñas, similar a lo que se encontró en otras regiones como Africa (16), islas del Caribe (17) e India (18).

No parece haber ninguna barrera epidemiológica insuperable para la erradicación de los casos de infección del pian en la provincia de Esmeraldas. No se ha demostrado la existencia de reservorio animal alguno en este continente o en este hemisferio (19). La erradicación del pian infeccioso requerirá de un mayor control y estudio de la enfermedad a nivel local o una campaña más agresiva de detección y tratamiento de nuevos casos, así como su control y vigilancia en las áreas endémicas. Aún más, la disponibilidad y el uso de jabón con énfasis en el aseo personal, ayudará mucho en la prevención del pian en las regiones rurales tropicales en la provincia de Esmeraldas.

\section{Agradecimientos}

El estudio fue financiado por la oficina local de la Organización Panamericana de la Salud, Quito, Ecuador y el Hospital Vozandes, Quito. Reconocemos la ayuda de los promotores de la salud del área, especialmente a los señores Gabriel Ayoui y Trifilo Ayoui.

\section{Referencias}

1. Antal GM. Global report on yaws and other endemic treponematoses. Southeastern Asian. J Trop Med Publ Hlth 1986;17(suppl):1-2.

2. St John RK. Yaws in Americas. Rev Infect Dis 1985;7(suppl 2):S266-72.

3. Naranjo MA. Pian en la provincia de Esmeraldas. Rev Ecuat Med Cien Biol 1977;13:179-82.
4. Ministerio de Salud Pública del Ecuador. Programa de control y vigilancia epidemiológica del pian, leishmaniasis y Hansen en la provincia de Esmeraldas. Dirección Nacional de Control y Vigilancia Epidemiológica, 1983.

5. Guderian RH, Dávalos A. Pian en el río Onzole, provincia de Esmeraldas. Medicina Ecuatoriana 1985;2:17-20.

6. Guderian RH, Guevara A, Dávalos A. La presencia de gangosa en el río Cayapas, provincia de Esmeraldas. Rev Ecuat Hig Med Trop 1987;37:53-60.

7. Guderian RH, Beck J, Stone DJ, Isabel K, Mackenzie CD. Onchocerciasis in Ecuador: recent observations in the province of Esmeraldas. J Trop Med Hyg 1988; 91:161-8.

8. Rumbea JG, Lazo RF, Cedeño JV. Consideraciones clínicas y epidemiológicas de la oncocercosis en poblaciones predominantes de colo de la provincia de Esmeraldas, Ecuador. Rev Ecuat Hig Med Trop 1980;33:17-31.

9. Perine PL, Hopkins DR, Nielmel PLA, St John RK, Causse G, Antal GM. En: Handbook of endemic treponematoses. Geneva: World Health Organizatión, 1984.

10. Larsen LA, D'Costa JF. Laboratory tests and serologic surveillance for yaws and conditions. Southeastern Asian. J Trop Med Publ Hlth 1986;17(suppl):70-7.

11. Vorst FA. Clinical diagnosis and changing manifestations of treponemal infections. Rev Infect Dis 1985;7(suppl. 2):S327-31.

12. World Health Organization. Treponemal infections. Tech Report Ser. Geneva: World Health Organization, 1982:674.

13. Santiana A. Pian. En: El estudio de la patología del oriente. Ministerio de la Comisión Sanitaria y Prevención Social, 1942.

14. Paltaw CJD. Pian. En: Observaciones sobre patología tropical. Ministerio de Gobierno, Quito, Ecuador, 1944.

15. Rodríguez UW. Yaws in Colombia. Rev Infect Dis 1985;17(suppl):70-7.

16. Ziefer A, Lanoie LO, Meyers MM, Vanderpas J, Charon F, Conner DH. Studies on a focus of yaws in Ubangi. Trop Med Parasit 1985;36:63-71.

17. Alemaena $\mathbf{O}$. Yaws situation in the Solomon islands. Southeast Asian J Trop Med Publ HIth 1986; 17(suppl):14-18.

18. Dutta M. Yaws in India. Southeast Asian J Trop Med Publ HIth 1986;17(suppl):35-41.

19. Frebourgh-Blane A, Mollaret HH. Natural treponematosis of the African primate. Primates Med 1969; 3:113-21. 